\title{
Case Report: Application of Acupuncture to Treat Knee Pain
}

\author{
Sherry Li, Elizabeth Piech, Bin Xu*, Josephine Fan* and Yemeng \\ Chen \\ New York College of Traditional Chinese Medicine, USA \\ ${ }^{*}$ Corresponding authors: Bin $\mathrm{Xu}$, New York College of Traditional Chinese Medicine, \\ Case Report \\ Volume 2 Issue 8 \\ Received Date: November 16, 2018 \\ Published Date: November 28, 2018 \\ DOI: $10.23880 /$ jonam-16000158
}

200 Old Country Road, Suite 500, Mineola, NY 11501, USA, Tel: (516) 739-1545; Email: ceu@nyctcm.edu

Josephine Fan, New York College of Traditional Chinese Medicine, 200 Old Country Road, Suite 500, Mineola, NY 11501, USA, Tel: (646) 582-9896; Email: jofanx@nyctcm.edu

\section{Abstract}

This case study presents an 86 year old female patient suffering severe pain resulting from knee osteoarthritis, seeking acupuncture as a treatment option to alleviate her symptoms. After four treatments, patient self-reported significant improvement in range of motion and decrease in pain, which allowed for higher quality of life. It should be noted that the patient is taking Plavix, an anticoagulant for those intolerant of aspirin, which has the reported side effect of joint pain. It is also possible that acupuncture is acting to decrease the medication side effects. Based on these results, acupuncture may be a viable option as a complementary mode of treatment for symptom management that warrants further investigation.

Keywords: Acupuncture; Osteoarthritis; Anti-Inflammatory; Traditional Chinese Medicine

\section{Introduction}

Knee Osteoarthritis (KOA) is one of the most common types of chronic arthritis and can cause articular cartilage damage, joint and para-knee articular soft tissues degeneration [1,2]. Approximately $60 \%$ of the male population and $70 \%$ of the female population of older adults in the United States suffer from KOA and the related difficulties in their activities. Decreased quality of life occurs in daily living, with lessened functional mobility in the community, home, and workplace environment due to the symptoms of joint pain, swelling, and deformity [2,3]. The American College of Rheumatology guidelines for treating KOA include several approaches used to alleviate pain including nonpharmacological modalities (such as surgery) and prescribed medications [4]. Non-steroidal antiinflammatory drugs are the most common drugs used for alleviating pain from KOA but they have the possible side effect of causing gastrointestinal bleeding [5]. Furthermore, elder adults often have co-morbidities which can easily increase the side effects due to drug interactions [1]. Acupuncture is an ancient Chinese medical modality which can be effectively used for treating various diseases and relieves symptoms and discomfort [6]. A systematic review done by the Osteoarthritis Research International Society indicated that patients with KOA can benefit from acupuncture treatment to reduce the symptoms of pain [7].

\section{Case History}

The female 86-year-old patient came to the Mineola teaching clinic of New York College of Traditional Chinese Medicine in March 2017 with severe pain in both of her knees caused by osteoarthritis. The arthritis was 


\section{Journal of Natural \& Ayurvedic Medicine}

diagnosed three years ago and the symptoms were increasing in severity. Her responses under the Visal Analog Sacle for pain were 8/10 for the right knee and $7 / 10$ for the left. The pain regions were focused primarily on the anterior of the knee joint and the shin. The patient stated that the discomfort occurs mostly when she has to stand or walk for even a short period of time, limiting her mobility at home and in the community. The patient also reported decreased quality of sleep due to pain at night. Patient had tried various treatment methods before coming to our acupuncture clinic. However, only ice packs and knee braces helped to alleviate the pain, but only for a very short period of time. The patient is on insulin for diabetes and Plavix as a blood thinner

\section{Musculoskeletal Evaluation}

The patient reported $8 / 10$ pain on the right knee and $7 / 10$ on the left knee, using a numeric one to ten pain rating scale. During the evaluation, the patient demonstrated limited active range of motion on both knees Including both knee flexions $45^{\circ}$ (Normal Values for Range of Motion of knee $130^{\circ}$ ) and extensions $40^{\circ}$ (Normal Values for Range of Motion of knee $120^{\circ}$ ) . Furthermore, the results of manual muscle test evaluation also indicated decreased muscle strength on both lower extremities.

\section{Testing and Diagnosis}

According to the notes from the patient's family physician, the patient has a medical history of diabetes and knee osteoarthritis.

\section{Assessment Using Traditional Chinese Medicine}

We utilized the four pillars of Traditional Chinese Medicine (TCM) to evaluation the patient's overall constitution and complexion. Based on the self-report from the patient, she demonstrated a tendency to be warm and preferred to drink cold water. The patient reported normal appetite but had bitter taste in her mouth. She had difficulty with defecation which occurred only four to five times every week. She described that she had a poor quality of sleep and could only sleep approximately five hours every night. She has constant knee pain on both sides and 9/10 pain scale on the right knee while $7 / 10$ on the left. The pain is aggravated by movement. Ice packs and knee bracing does seem to help alleviate knee pain for a short period of time. The patient's tongue was red with a scanty moist coating. Her pulse was deep soft on the right side and deep wiry on the left side. The final TCM diagnosis was Kidney essence deficiency.

\section{Treatment Plan}

Based on the TCM diagnosis, the treatment plan was to alleviate the patient's pain by notifying the Kidney and boosting Kidney essence. The long term goal of the intervention sessions was to reduce the knee pain and to increase the range of motion on both lower extremities including active range of motion on both knees including both knee flexions $100^{\circ}$ (Normal Values for Range of Motion of knee $130^{\circ}$ ) and extensions $110^{\circ}$ (Normal Values for Range of Motion of knee $120^{\circ}$ ). According to the theory of Liu's Scalp Acupuncture, the scalp knee points were selected to reduce the pain on both knees. Based on the TCM treatment principle, CV 23, ST 29, KI 3, Master Tung's points of Lower Yellow $(77.17 ; 77.18$; 77.19; 77.20)and Upper Yellow (88.12; 88.13;88.14 )and Umbilical Kidney, Heart, Liver were utilized as well. Patient was positioned in supine on treatment table with both knees supported for thirty minutes during the treatment session. For the follow-up treatment sessions, the scalp knee points and umbilical acupuncture points were remained for needling, some other points were added such as LI 11, GB 20, and ST 29.

\section{Treatment Results}

After four treatments, the patient reported the pain on the right knee had decreased from $9 / 10$ to $7 / 10$ on a 10-point numerical pain scale, while the pain on left knee had decreased from $7 / 10$ to $4 / 10$. The patient also demonstrated significant improvement on range of motion of both lower extremities. She reported that with decreased joint pain, she was able to participate more in her daily routine activities and demonstrated more independence and improved quality of life.

\section{Discussion}

There has been an increased interest in acupuncture as alternative to traditional treatment methods for musculoskeletal pain, including the symptoms related to osteoarthritis and research into the mechanisms of how it works have been undertaken. The analgesic properties of acupuncture stimulation has now been speculated to at least be correlated with ERK (extracellular signalregulated kinase) phosphorylation following acupuncture needling and therefore could be why patients report decrease in their pain symptoms. In addition, improved microcirculation coupled with analgesic effects of needle stimulation could bring about long term improvements. Given the self-reported improvement the patient has experienced, acupuncture may be an option for those suffering from arthritis who wish to reduce their reliance 


\section{Journal of Natural \& Ayurvedic Medicine}

on medication [8-9]. One particular issue of note is that the patient is on Plavix, an anticoagulant prescribed to those who are unable to take aspirin as the standard blood thinner (either due to allergy or contradicting drug interactions), which is listed with the side effect of joint pain, and in some instances, the uncommon side effect of acute osteoarthritis. While the patient may not be able to stop taking Plavix, acupuncture could be a viable, nonpharmacological alternative for managing the side effect.he female 86-year-old patient came to the New York College of Traditional Chinese Medicine Mineola teaching clinic in March 2017 with severe pain in both of her knees caused by osteoarthritis. The arthritis was diagnosed three years ago and the symptoms were increasing in severity. Her responses under the Ten Point Pain Scale were $8 / 10$ for the right knee and $7 / 10$ for the left. The pain regions were focused primarily on the anterior of the knee joint and the shin. The patient stated that the discomfort occurs mostly when she has to stand or walk for even a short period of time, limiting her mobility at home and in the community. The patient also reported decreased quality of sleep due to pain at night.

Patient had tried various treatment methods before coming to our acupuncture clinic. However, only ice packs and knee braces helped to alleviate the pain, but only for a very short period of time.

The patient is on insulin for diabetes and Plavix as a blood thinner.

\section{References}

1. Helianthi DR, Simadibrata C, Srilestari A, Wahyudi ER, Hidayat R (2016) Pain Reduction After Laser Acupuncture Treatment in Geriatric Patients with Knee Osteoarthritis: a Randomized Controlled Trial. Acta Med Indones 48(2): 114-121.
2. Ding, Y, Wang Y, Shi X, Luo Y, Gao Y, et al. (2016) Effect of ultrasound-guided acupotomy vs electroacupuncture on knee osteoarthritis: a randomized controlled study. J Tradit Chin Med 36(4): 450-455.

3. Sarzi-Puttini P, Cimmino MA, Scarpa R, Caporali R, Parazzini F, et al. (2005) Osteoarthritis: an overview of the disease and its treatment strategies. Semin arthritis Rheum 35(1): 1-10.

4. Gamble R (2000) Recommendations for the medical management of osteoarthritis of the hip and knee. Arthritis Rheum 43(9): 1905-1915.

5. Tramèr MR, Moore RA, Reynolds DJ, McQuay HJ (2000) Quantitative estimation of rare adverse events which follow a biological progression: a new model applied to chronic NSAID use. Pain 85(1): 169-182.

6. Christensen PA, Noreng M, Andersen PE, Nielsen JW (1989) Electroacupuncture and postoperative pain. BJA: British Journal of Anaesthesia 62(3): 258-262.

7. Zhang W, Moskowitz RW, Nuki G, Abramson S, Altman $\mathrm{RD}$, et al. (2008) OARSI recommendations for the management of hip and knee osteoarthritis. Part II: OARSI evidence-based. Expert consensus guideline. Osteoarthritis Cartilage 16(2): 137-162.

8. Park J, Park JJ, Jeon S, Doo A, Kim S, et al. (2014) From Peripheral to Central: The Role of ERK Signaling Pathway in Acupuncture Analgesia. J Pain 15(5): 535549.

9. Min S, Lee H, Kim S, Park J, Chae Y, et al. (2015) Local Changes in Microcirculation and the Analgesic Effects of Acupuncture: A Laser Doppler Perfusion Imaging Study. J Altern Complement Med 21(1): 46-52. 